\title{
ON UNIQUENESS OF ENTROPY SOLUTIONS FOR NONLINEAR ELLIPTIC DEGENERATE ANISOTROPIC EQUATIONS
}

\begin{abstract}
Yu. S. Gorban. On uniqueness of entropy solutions for nonlinear elliptic degenerate anisotropic equations, Mat. Stud. 47 (2017), 59-70.

In the present paper we deal with the Dirichlet problem for a class of degenerate anisotropic elliptic second-order equations with $L^{1}$-right-hand sides in a bounded domain of $\mathbb{R}^{n}(n \geqslant 2)$. This class is described by the presence of a set of exponents $q_{1}, \ldots, q_{n}$ and a set of weighted functions $\nu_{1}, \ldots, \nu_{n}$ in growth and coercitivity conditions on coefficients of the equations. The exponents $q_{i}$ characterize the rates of growth of the coefficients with respect to the corresponding derivatives of unknown function, and the functions $\nu_{i}$ characterize degeneration or singularity of the coefficients with respect to independent variables. Our aim is to study the uniqueness of entropy solution of the problem under consideration.
\end{abstract}

Introduction. The studying of nonlinear elliptic second-order equations with $L^{1}$-data and measures as data is one of the important directions of a modern differential equation theory. In this theory the concepts of a weak solution, entropy solution, renormalized solution were introduced, the theorems on existence and uniqueness of these solutions were proved, and their belonging to Lebesgue and Sobolev spaces were established.

A weak solution (solution from $W^{1,1}$ in sense of the integral identity for smooth functions) to equations with $L^{1}$-right-hand sides is a natural analogue of a generalized solution to equations with "well enough", right-hand sides. The theorems on the existence of a weak solution to the Dirichlet problem for nonlinear elliptic equations were obtained in [5], [6]. Remark that a weak solution exists not for all values of a parameter characterizing the growth of equation's coefficients with respect to the corresponding derivatives of unknown function. In general, a weak solution is not a unique one.

An effective approach to the solvability of the Dirichlet problem for nonlinear elliptic second-order equations with $L^{1}$-right-hand sides has been proposed in [4]. There a concept of an entropy solution to the problem under consideration was introduced. This solution belongs to a new special function's class that includes the corresponding Sobolev space. Under standard growth, coercitivity and strict monotonicity conditions on the equation's coefficients authors proved the theorem on existence and uniqueness of an entropy solution to the given problem. Notice that an entropy solution is unique for all values of a parameter characterizing the growth of equation's coefficients with respect to the corresponding derivatives of unknown function.

2010 Mathematics Subject Classification: 35J25, 35J60, 35J70.

Keywords: Nonlinear elliptic degenerate anisotropic second-order equations; $L^{1}$-data; Dirichlet problem; uniqueness of entropy solution.

doi:10.15330/ms.47.1.59-70

(C) Yu. S. Gorban, 2017 
Above-mentioned works and other close investigations are devoted to $L^{1}$-theory for nonlinear equations with isotropic and nondegenerate (with respect to the independent variables) coefficients. As for the solvability of nonlinear elliptic second-order equations with anisotropy and degeneracy (with respect to the independent variables), we note the following works. The existence of a weak solution to the Dirichlet problem for a model nondegenerate anisotropic equation with right-hand side measure was established in [7]. The existence of weak solutions for a class of nondegenerate anisotropic equations with locally integrable data was proved in [3]. Solvability of the Dirichlet problem for degenerate isotropic equations with $L^{1}$-data and measures as data was studied in [1], [2], [8], [9], [16]. Remark that in [1], [8], the existence of entropy solutions to the given problem was proved in the case of $L^{1}$-data, and in [2], the existence of a renormalized solution of the problem for the same case was established. In [2], [9], [16], the existence of distributional solutions of the problem was obtained in the case of right-hand side measures.

Solvability of the Dirichlet problem for a class of degenerate anisotropic elliptic secondorder equations with $L^{1}$-right-hand sides was studied in [14]. This class is described by the presence of a set of exponents $q_{1}, \ldots, q_{n}$ and of a set of weighted functions $\nu_{1}, \ldots, \nu_{n}$ in growth and coercitivity conditions on coefficients of the equations under consideration. The exponents $q_{i}$ characterize the rates of growth of the coefficients with respect to the corresponding derivatives of unknown function, and the functions $\nu_{i}$ characterize degeneration or singularity of the coefficients with respect to the independent variables.

In [14], the theorem on the existence and uniqueness of entropy solution to the Dirichlet for this class of the equations was proved (see [14], Theorem 3.2). Observe that the proof of this theorem is based on use of some results of [11]-[13] on the existence and properties of solutions of second-order variational inequalities with $L^{1}$-right-hand sides and sufficiently general constraints. Note that in [11]-[14] right-hand sides to the investigated variational inequalities and equations depend on independent variables only, and belong to the class $L^{1}$.

The present paper is devoted to the Dirichlet problem for the same class of the nonlinear elliptic second-order equations in divergence form with degenerate anisotropic coefficients as in [14]. Now right-hand sides to the given equations depend on independent variables and unknown function. In our case we have no opportunity to use the results [11]-[13] directly. We follow a general approach for proving the main result of this work (theorem 1). As we mentioned above, this approach has been proposed in [4] to the investigation on the existence and properties of solutions for nonlinear elliptic second-order equations with isotropic nondegenerate (with respect to the independent variables) coefficients and $L^{1}$-righthand sides. In [11], [13] this approach has been taken to the anisotropic degenerate case. Also we use some ideas of [15].

1. Preliminaries. In this section we give some results of [13] which will be used in the sequel.

Let $n \in \mathbb{N}, n \geqslant 2, \Omega$ be a bounded domain in $\mathbb{R}^{n}$ with the boundary $\partial \Omega$, and let for every $i \in\{1, \ldots, n\}$ we have $q_{i} \in(1, n)$.

We set $q=\left\{q_{i}: i=1, \ldots, n\right\}$,

$$
\bar{q}=\left(\frac{1}{n} \sum_{i=1}^{n} \frac{1}{q_{i}}\right)^{-1}, \quad \hat{q}=\frac{n(\bar{q}-1)}{(n-1) \bar{q}} .
$$

Let for every $i \in\{1, \ldots, n\} \quad \nu_{i}$ be a nonnegative function on $\Omega$ such that $\nu_{i}>0$ a.e. 
in $\Omega$,

$$
\nu_{i} \in L_{\mathrm{loc}}^{1}(\Omega), \quad\left(1 / \nu_{i}\right)^{1 /\left(q_{i}-1\right)} \in L^{1}(\Omega) .
$$

We set $\nu=\left\{\nu_{i}: i=1, \ldots, n\right\}$. We denote by $W^{1, q}(\nu, \Omega)$ the set of all functions $u \in$ $W^{1,1}(\Omega)$ such that for every $i \in\{1, \ldots, n\}$ we have $\nu_{i}\left|D_{i} u\right|^{q_{i}} \in L^{1}(\Omega)$.

Let $\|\cdot\|_{1, q, \nu}$ be the mapping from $W^{1, q}(\nu, \Omega)$ into $\mathbb{R}$ such that for every function $u \in$ $W^{1, q}(\nu, \Omega)$

$$
\|u\|_{1, q, \nu}=\int_{\Omega}|u| d x+\sum_{i=1}^{n}\left(\int_{\Omega} \nu_{i}\left|D_{i} u\right|^{q_{i}} d x\right)^{1 / q_{i}} .
$$

The mapping $\|\cdot\|_{1, q, \nu}$ is a norm in $W^{1, q}(\nu, \Omega)$, and, in view of the second inclusion of (1), the set $W^{1, q}(\nu, \Omega)$ is a Banach space with respect to the norm $\|\cdot\|_{1, q, \nu}$. Moreover, by virtue of the first inclusion of $(1)$, we have $C_{0}^{\infty}(\Omega) \subset W^{1, q}(\nu, \Omega)$.

We denote by $\stackrel{\circ}{W}^{1, q}(\nu, \Omega)$ the closure of the set $C_{0}^{\infty}(\Omega)$ in space $W^{1, q}(\nu, \Omega)$. Evidently, the set $\stackrel{\circ}{W}^{1, q}(\nu, \Omega)$ is a Banach space with respect to the norm induced by the norm $\|\cdot\|_{1, q, \nu}$. It is obvious that $\stackrel{\circ}{W}^{1, q}(\nu, \Omega) \subset \stackrel{\circ}{W}^{1,1}(\Omega)$.

Further, let for every $k>0 \quad T_{k}: \mathbb{R} \rightarrow \mathbb{R}$ be the function such that

$$
T_{k}(s)= \begin{cases}s, & \text { if }|s| \leqslant k, \\ k \operatorname{sign} s, & \text { if }|s|>k .\end{cases}
$$

By analogy with known results for nonweighted Sobolev spaces (see for instance [10]) we have: if $u \in \stackrel{\circ}{W}{ }^{1, q}(\nu, \Omega)$, and $k>0$, then $T_{k}(u) \in \stackrel{\circ}{W}^{1, q}(\nu, \Omega)$, and for every $i \in\{1, \ldots, n\}$,

$$
D_{i} T_{k}(u)=D_{i} u \cdot 1_{\{|u|<k\}} \text { a. e. in } \Omega \text {. }
$$

We denote by $\stackrel{\circ}{\mathcal{T}}^{1, q}(\nu, \Omega)$ the set of all functions $u: \Omega \rightarrow \mathbb{R}$ such that for every $k>0$ $T_{k}(u) \in \stackrel{\circ}{W}^{1, q}(\nu, \Omega)$. Clearly, $\stackrel{\circ}{W}^{1, q}(\nu, \Omega) \subset \stackrel{\circ}{\mathcal{T}}^{1, q}(\nu, \Omega)$.

For every $u: \Omega \rightarrow \mathbb{R}$ and for every $x \in \Omega$ we set $k(u, x)=\min \{l \in \mathbb{N}:|u(x)| \leqslant l\}$.

Definition 1. Let $u \in \stackrel{\circ}{\mathcal{T}}^{1, q}(\nu, \Omega)$, and $i \in\{1, \ldots, n\}$. Then $\delta_{i} u: \Omega \rightarrow \mathbb{R}$ is the function such that for every $x \in \Omega \quad \delta_{i} u(x)=D_{i} T_{k(u, x)}(u)(x)$.

Definition 2. If $u \in \stackrel{\circ}{\mathcal{T}}^{1, q}(\nu, \Omega)$, then $\delta u: \Omega \rightarrow \mathbb{R}^{n}$ is the mapping such that for every $x \in \Omega$ and for every $i \in\{1, \ldots, n\} \quad(\delta u(x))_{i}=\delta_{i} u(x)$.

Now we give several propositions which will be used in the next sections.

Proposition 1. Let $u \in \stackrel{\circ}{\mathcal{T}}^{1, q}(\nu, \Omega)$. Then for every $k>0$ we have $D_{i} T_{k}(u)=\delta_{i} u \cdot 1_{\{|u|<k\}}$ a.e. in $\Omega, i=1, \ldots, n$.

Note that for every function $u \in \stackrel{\circ}{W^{1}, q}(\nu, \Omega) \quad \delta_{i} u=D_{i} u$ a.e. in $\Omega, \quad i=1, \ldots, n$.

Proposition 2. Let $u \in \stackrel{\circ}{\mathcal{T}}^{1, q}(\nu, \Omega), w \in \stackrel{\circ}{W}^{1, q}(\nu, \Omega) \cap L^{\infty}(\Omega)$. Then $u-w \in \stackrel{\circ}{\mathcal{T}}^{1, q}(\nu, \Omega)$, and for every $i \in\{1, \ldots, n\}$ and for every $k>0$ we have

$$
D_{i} T_{k}(u-w)=\delta_{i} u-D_{i} w \quad \text { a.e. in }\{|u-w|<k\} .
$$


Proposition 3. There exists a positive constant $c_{0}$ depending only on $n, q,\left\|1 / \nu_{i}\right\|_{L^{1 /\left(q_{i}-1\right)}(\Omega)}$, $i=1, \ldots, n$, such that for every function $u \in \stackrel{\circ}{W^{1, q}}(\nu, \Omega)$

$$
\left(\int_{\Omega}|u|^{n /(n-1)} d x\right)^{(n-1) / n} \leqslant c_{0} \prod_{i=1}^{n}\left(\int_{\Omega} \nu_{i}\left|D_{i} u\right|^{q_{i}} d x\right)^{1 / n q_{i}} .
$$

2. Statement of the Dirichlet problem. The concept of its entropy solution. Let $c_{1}, c_{2}>0, g_{1}, g_{2} \in L^{1}(\Omega), g_{1}, g_{2} \geqslant 0$ in $\Omega$, and let for every $i \in\{1, \ldots, n\} \quad a_{i}: \Omega \times \mathbb{R}^{n} \rightarrow \mathbb{R}$ be a Carathéodory function. We suppose that for almost every $x \in \Omega$ and for every $\xi \in \mathbb{R}^{n}$,

$$
\begin{gathered}
\sum_{i=1}^{n}\left(1 / \nu_{i}\right)^{1 /\left(q_{i}-1\right)}(x)\left|a_{i}(x, \xi)\right|^{q_{i} /\left(q_{i}-1\right)} \leqslant c_{1} \sum_{i=1}^{n} \nu_{i}(x)\left|\xi_{i}\right|^{q_{i}}+g_{1}(x), \\
\sum_{i=1}^{n} a_{i}(x, \xi) \xi_{i} \geqslant c_{2} \sum_{i=1}^{n} \nu_{i}(x)\left|\xi_{i}\right|^{q_{i}}-g_{2}(x) .
\end{gathered}
$$

Moreover, we assume that for almost every $x \in \Omega$ and for every $\xi, \xi^{\prime} \in \mathbb{R}^{n}, \xi \neq \xi^{\prime}$,

$$
\sum_{i=1}^{n}\left[a_{i}(x, \xi)-a_{i}\left(x, \xi^{\prime}\right)\right]\left(\xi_{i}-\xi_{i}^{\prime}\right)>0
$$

Now we give one result of [14] which will be used in the sequel.

Proposition 4. The following assertions hold:

a) if $u \in \stackrel{\circ}{\mathcal{T}}^{1, q}(\nu, \Omega), w \in \stackrel{\circ}{W^{1, q}}(\nu, \Omega) \cap L^{\infty}(\Omega), k>0, l \geqslant k+\|w\|_{L^{\infty}(\Omega)}$, and $i \in\{1, \ldots, n\}$, then $a_{i}(x, \delta u) D_{i} T_{k}(u-w)=a_{i}\left(x, \nabla T_{l}(u)\right) D_{i} T_{k}(u-w)$ a.e. in $\Omega$;

b) if $u \in \stackrel{\circ}{\mathcal{T}}^{1, q}(\nu, \Omega), w \in \stackrel{\circ}{W^{1, q}}(\nu, \Omega) \cap L^{\infty}(\Omega), k>0$, and $i \in\{1, \ldots, n\}$, then $a_{i}(x, \delta u) D_{i} T_{k}(u-w) \in L^{1}(\Omega)$.

Let $F: \Omega \times \mathbb{R} \rightarrow \mathbb{R}$ be a Carathéodory function. We consider the following Dirichlet problem:

$$
\begin{gathered}
-\sum_{i=1}^{n} \frac{\partial}{\partial x_{i}} a_{i}(x, \nabla u)=F(x, u) \quad \text { in } \Omega, \\
u=0 \quad \text { on } \partial \Omega .
\end{gathered}
$$

Definition 3. An entropy solution of problem (6), (7) is a function $u \in \stackrel{\circ}{\mathcal{T}}^{1, q}(\nu, \Omega)$ such that:

$$
F(x, u) \in L^{1}(\Omega)
$$

for every function $w \in \stackrel{\circ}{W^{1, q}}(\nu, \Omega) \cap L^{\infty}(\Omega)$ and for every $k \geqslant 1$,

$$
\int_{\Omega}\left\{\sum_{i=1}^{n} a_{i}(x, \delta u) D_{i} T_{k}(u-w)\right\} d x \leqslant \int_{\Omega} F(x, u) T_{k}(u-w) d x .
$$

Note that the left-hand integral in (9) is finite. It follows from assertion b) of Proposition 4 . The right-hand integral in (9) is also finite. It follows from the boundedness of the function $T_{k}$ and inclusion (8). 
3. On uniqueness of an entropy solution. Firstly, we prove two auxiliary results.

Lemma 1. Let $u$ be an entropy solution of the Dirichlet problem (6), (7). Then there exists a nonnegative constant $M$ such that for every $k \geqslant 1$,

$$
\text { meas }\{|u| \geqslant k\} \leqslant M k^{-\hat{q}} \text {. }
$$

Proof. We fix an arbitrary function $v \in \stackrel{\circ}{W}^{1, q}(\nu, \Omega) \cap L^{\infty}(\Omega)$, and set

$$
\begin{gathered}
M_{*}=\frac{2}{c_{2}}\left\{\frac{c_{2}}{2 c_{1}}\left\|g_{1}\right\|_{L^{1}(\Omega)}+\left\|g_{2}\right\|_{L^{1}(\Omega)}+(2 n)^{n-1}\left(\frac{c_{1}}{c_{2}}+1\right)^{n-1} \int_{\Omega}\left\{\sum_{i=1}^{n} \nu_{i}\left|D_{i} v\right|^{q_{i}}\right\} d x+\right. \\
\left.+\left(1+\|v\|_{L^{\infty}(\Omega)}\right) \int_{\Omega}|F(x, u)| d x\right\}, \quad M=\left(c_{0} M_{*}^{1 / \bar{q}}\right)^{n /(n-1)} .
\end{gathered}
$$

Let $k \geqslant 1$. We put $k_{1}=k+\|v\|_{L^{\infty}(\Omega)}$,

$$
I=\sum_{i=1}^{n} \int_{\left\{|u-v|<k_{1}\right\}} \nu_{i}\left|\delta_{i} u\right|^{q_{i}} d x, \quad J=\sum_{i=1}^{n} \int_{\left\{|u-v|<k_{1}\right\}}\left|a_{i}(x, \delta u)\right|\left|D_{i} v\right| d x .
$$

In view of $(9)$,

$$
\int_{\Omega}\left\{\sum_{i=1}^{n} a_{i}(x, \delta u) D_{i} T_{k_{1}}(u-v)\right\} d x \leqslant \int_{\Omega} F(x, u) T_{k_{1}}(u-v) d x .
$$

Using Propositions 1 and 2, and (4), we obtain from this inequality:

$$
c_{2} I \leqslant k_{1} \int_{\Omega}|F(x, u)| d x+\left\|g_{2}\right\|_{L^{1}(\Omega)}+J .
$$

On the other hand, taking into account Young's inequality and (3), we find that

$$
J \leqslant \frac{c_{2}}{2} I+\frac{c_{2}}{2 c_{1}}\left\|g_{1}\right\|_{L^{1}(\Omega)}+(2 n)^{n-1}\left(\frac{c_{1}}{c_{2}}+1\right)^{n-1} \int_{\Omega}\left\{\sum_{i=1}^{n} \nu_{i}\left|D_{i} v\right|^{q_{i}}\right\} d x .
$$

From latter two estimates it follows that

$$
I \leqslant M_{*} k .
$$

Further, we have $\left|T_{k}(u)\right|=k$ on $\{|u| \geqslant k\}$. Then

$$
k^{n /(n-1)} \text { meas }\{|u| \geqslant k\} \leqslant \int_{\Omega}\left|T_{k}(u)\right|^{n /(n-1)} d x .
$$

Since $T_{k}(u) \in \stackrel{\circ}{W}^{1, q}(\nu, \Omega)$, from (11), Propositions 3 and 1 we get

$$
\begin{aligned}
&\left(\int_{\Omega}\left|T_{k}(u)\right|^{n /(n-1)} d x\right)^{(n-1) / n} \leqslant c_{0} \prod_{i=1}^{n}\left(\int_{\Omega} \nu_{i}\left|D_{i} T_{k}(u)\right|^{q_{i}} d x\right)^{1 / n q_{i}}= \\
&= c_{0} \prod_{i=1}^{n}\left(\int_{\{|u|<k\}} \nu_{i}\left|\delta_{i} u\right|^{q_{i}} d x\right)^{1 / n q_{i}} \leqslant c_{0} \prod_{i=1}^{n}\left(\int_{\left\{|u-v|<k_{1}\right\}} \nu_{i}\left|\delta_{i} u\right|^{q_{i}} d x\right)^{1 / n q_{i}} \leqslant \\
& \leqslant c_{0} I^{1 / \bar{q}} \leqslant c_{0}\left(M_{*} k\right)^{1 / \bar{q}} .
\end{aligned}
$$

This estimate and (12) imply (10). 
Lemma 2. Let $u$ be an entropy solution of the Dirichlet problem (6), (7). Then for every $v \in \stackrel{\circ}{W}^{1, q}(\nu, \Omega) \cap L^{\infty}(\Omega), k \geqslant 1, h \geqslant 1$,

$$
\begin{gathered}
\int_{\{h \leqslant|u-v|<h+k\}}\left\{\sum_{i=1}^{n} \nu_{i}\left|\delta_{i} u\right|^{q_{i}}\right\} d x \leqslant \frac{2 k}{c_{2}} \int_{\{|u-v| \geqslant h\}}|F(x, u)| d x+ \\
+\frac{2(2 n)^{n-1}}{c_{2}}\left(\frac{c_{1}}{c_{2}}+1\right)^{n-1} \int_{\{h \leqslant|u-v|<h+k\}}\left\{\sum_{i=1}^{n} \nu_{i}\left|D_{i} v\right|^{q_{i}}+g_{1}+g_{2}\right\} d x .
\end{gathered}
$$

Proof. We fix arbitrary $v \in \stackrel{\circ}{W}^{1, q}(\nu, \Omega) \cap L^{\infty}(\Omega), k \geqslant 1$, and $h \geqslant 1$.

Put

$$
w=v+T_{h}(u-v), \quad k_{1}=k+\|w\|_{L^{\infty}(\Omega)} .
$$

From (9) and assertion a) of Proposition 4 it follows that

$$
\int_{\{|u-w|<k\}}\left\{\sum_{i=1}^{n} a_{i}\left(x, \nabla T_{k_{1}}(u)\right) D_{i} T_{k}(u-w)\right\} d x \leqslant k \int_{\{|u-v| \geqslant h\}}|F(x, u)| d x .
$$

We set $G_{1}=\{h \leqslant|u-v|<h+k\}, G_{2}=\{|u-v|<h\}$. Observe that

$$
\{|u-w|<k\}=G_{1} \cup G_{2}, \quad G_{1} \cap G_{2}=\varnothing .
$$

We have

$$
T_{k}(u-w)=T_{k_{1}}(u)-v-T_{h}(u-v) \quad \text { a.e. in } G_{1}, \quad T_{k}(u-w)=0 \quad \text { in } G_{2} .
$$

Hence, for every $i \in\{1, \ldots, n\}$,

$$
D_{i} T_{k}(u-w)=D_{i} T_{k_{1}}(u)-D_{i} v \quad \text { a.e. in } G_{1}, \quad D_{i} T_{k}(u-w)=0 \quad \text { a.e. in } \quad G_{2} .
$$

These facts, and (14), (15) imply

$$
\begin{gathered}
\int_{G_{1}}\left\{\sum_{i=1}^{n} a_{i}\left(x, \nabla T_{k_{1}}(u)\right) D_{i} T_{k_{1}}(u)\right\} d x \leqslant \\
\leqslant \int_{G_{1}}\left\{\sum_{i=1}^{n} a_{i}\left(x, \nabla T_{k_{1}}(u)\right) D_{i} v\right\} d x+k \int_{\{|u-v| \geqslant h\}}|F(x, u)| d x .
\end{gathered}
$$

We denote by $I_{1}$ the integral from the left-hand side of (16), and by $I_{2}$ the integral from the right-hand side of (16). By virtue of (4), we get

$$
I_{1} \geqslant c_{2} \int_{G_{1}}\left\{\sum_{i=1}^{n} \nu_{i}\left|D_{i} T_{k_{1}}(u)\right|^{q_{i}}\right\} d x-\int_{G_{1}} g_{2} d x .
$$

From this estimate and (16) it follows that

$$
c_{2} \int_{G_{1}}\left\{\sum_{i=1}^{n} \nu_{i}\left|D_{i} T_{k_{1}}(u)\right|^{q_{i}}\right\} d x \leqslant k \int_{\{|u-v| \geqslant h\}}|F(x, u)| d x+\int_{G_{1}} g_{2} d x+I_{2} .
$$


Using (3) and Young's inequality, we obtain

$$
\begin{aligned}
I_{2} \leqslant & \frac{c_{2}}{2} \int_{G_{1}}\left\{\sum_{i=1}^{n} \nu_{i}\left|D_{i} T_{k_{1}}(u)\right|^{q_{i}}\right\} d x+\frac{c_{2}}{2 c_{1}} \int_{G_{1}} g_{1} d x+ \\
& +(2 n)^{n-1}\left(\frac{c_{1}}{c_{2}}+1\right)^{n-1} \int_{G_{1}}\left\{\sum_{i=1}^{n} \nu_{i}\left|D_{i} v\right|^{q_{i}}\right\} d x
\end{aligned}
$$

Note that in view of proposition 1 for every $i \in\{1, \ldots, n\}$ we have $D_{i} T_{k_{1}}(u)=\delta_{i} u$ a.e. in $G_{1}$. Taking into account this fact, we deduce the inequality (13) from (17) and (18).

Next theorem is the main result of this paper.

Theorem 1. Let for a.e. $x \in \Omega \quad F(x, \cdot)$ be the nonincreasing function on $\mathbb{R}$, and let $u_{1}, u_{2}$ be entropy solutions of the Dirichlet problem (6), (7). Then $u_{1}=u_{2}$ a.e. in $\Omega$.

Proof. We denote by $c_{i}, i=3,4, \ldots$, the positive constants depending only on $n, c_{1}$ and $c_{2}$.

Fix an arbitrary function $v \in \stackrel{\circ}{W}{ }^{1, q}(\nu, \Omega) \cap L^{\infty}(\Omega)$, and set

$$
\Phi_{j}=\sum_{i=1}^{n} \nu_{i}\left|D_{i} v\right|^{q_{i}}+g_{1}+g_{2}+\left|F\left(x, u_{j}\right)\right|, \quad j=1,2 .
$$

From Lemma 2 it follows that for every $k \geqslant 1, h \geqslant k+1$,

$$
\int_{\left\{h-k \leqslant\left|u_{j}-v\right|<h+k\right\}}\left\{\sum_{i=1}^{n} \nu_{i}\left|\delta_{i} u_{j}\right|^{q_{i}}\right\} d x \leqslant c_{3} k \int_{\left\{\left|u_{j}-v\right| \geqslant h-k\right\}} \Phi_{j} d x, \quad j=1,2 .
$$

Fix an arbitrary $k \geqslant 1, h \geqslant k+1$, and put

$$
\begin{gathered}
G=\left\{\left|u_{1}-u_{2}\right|<k,\left|u_{1}-v\right|<h,\left|u_{2}-v\right|<h\right\}, \quad G_{1}=\left\{\left|u_{1}-v\right|<h,\left|u_{2}-v\right|<h\right\}, \\
G_{2}=\left\{\left|u_{1}-v\right| \geqslant h\right\} \cup\left\{\left|u_{2}-v\right| \geqslant h\right\}, \quad w=v+T_{h}\left(u_{2}-v\right), \quad l=k+\|w\|_{L^{\infty}(\Omega)} .
\end{gathered}
$$

By virtue of Definition 3 and assertion a) of Proposition 4, we have

$$
\begin{gathered}
\int_{\Omega}\left\{\sum_{i=1}^{n} a_{i}\left(x, \delta u_{1}\right) D_{i} T_{k}\left(u_{1}-w\right)\right\} d x=\int_{\Omega}\left\{\sum_{i=1}^{n} a_{i}\left(x, \nabla T_{l}\left(u_{1}\right)\right) D_{i} T_{k}\left(u_{1}-w\right)\right\} d x \leqslant \\
\leqslant \int_{G_{1}} F\left(x, u_{1}\right) T_{k}\left(u_{1}-u_{2}\right) d x+k \int_{G_{2}}\left|F\left(x, u_{1}\right)\right| d x
\end{gathered}
$$

Now we estimate lower bound the left-hand side of this inequality. Put

$$
E^{\prime}=\left\{\left|u_{1}-w\right|<k,\left|u_{2}-v\right|<h\right\}, \quad E^{\prime \prime}=\left\{\left|u_{1}-w\right|<k,\left|u_{2}-v\right| \geqslant h\right\} .
$$

It is clear that

$$
G \subset E^{\prime}
$$

Besides, we have

$$
E^{\prime} \backslash G \subset\left\{h \leqslant\left|u_{1}-v\right|<h+k\right\} \cap\left\{h-k \leqslant\left|u_{2}-v\right|<h\right\},
$$




$$
E^{\prime \prime} \subset\left\{h-k \leqslant\left|u_{1}-v\right|<h+k\right\} .
$$
Hence,

In fact, let $x \in E^{\prime} \backslash G$. Then $\left|u_{1}(x)-u_{2}(x)\right|<k,\left|u_{2}(x)-v(x)\right|<h,\left|u_{1}(x)-v(x)\right| \geqslant h$.

$$
h \leqslant\left|u_{1}(x)-v(x)\right| \leqslant\left|u_{1}(x)-u_{2}(x)\right|+\left|u_{2}(x)-v(x)\right|<k+\left|u_{2}(x)-v(x)\right|<h+k .
$$

Inclusion (22) follows from these estimates. Let now $x \in E^{\prime \prime}$. Therefore,

$$
\left|u_{1}(x)-w(x)\right|<k, \quad\left|u_{2}(x)-v(x)\right| \geqslant h .
$$

By virtue of the second inequality of (24), and definition of the function $w$ we have $w(x)=v(x)+h \operatorname{sign}\left(u_{2}(x)-v(x)\right)$. So, in view of the first inequality of $(24)$, we get

$$
\begin{gathered}
\left|u_{1}(x)-v(x)\right| \leqslant\left|u_{1}(x)-w(x)\right|+|w(x)-v(x)|<h+k, \\
h=|v(x)-w(x)| \leqslant\left|u_{1}(x)-v(x)\right|+\left|u_{1}(x)-w(x)\right|<\left|u_{1}(x)-v(x)\right|+k .
\end{gathered}
$$

Hence, inclusion (23) is true.

Further, since

$$
T_{k}\left(u_{1}-w\right)=T_{l}\left(u_{1}\right)-T_{l}\left(u_{2}\right) \text { a.e. in } E^{\prime},
$$

for every $i \in\{1, \ldots, n\}$ we have

$$
D_{i} T_{k}\left(u_{1}-w\right)=D_{i} T_{l}\left(u_{1}\right)-D_{i} T_{l}\left(u_{2}\right) \text { a.e. in } E^{\prime} .
$$

By analogy,

$$
T_{k}\left(u_{1}-w\right)=T_{l}\left(u_{1}\right)-v-T_{h}\left(u_{2}-v\right) \text { a.e. in } E^{\prime \prime},
$$

thus, for every $i \in\{1, \ldots, n\}$ we have

$$
D_{i} T_{k}\left(u_{1}-w\right)=D_{i} T_{l}\left(u_{1}\right)-D_{i} v \text { a.e. in } E^{\prime \prime} .
$$

Taking into account (25) and (26), we obtain

$$
\begin{gathered}
\int_{\Omega}\left\{\sum_{i=1}^{n} a_{i}\left(x, \nabla T_{l}\left(u_{1}\right)\right) D_{i} T_{k}\left(u_{1}-w\right)\right\} d x= \\
=\int_{E^{\prime}}\left\{\sum_{i=1}^{n} a_{i}\left(x, \nabla T_{l}\left(u_{1}\right)\right)\left[D_{i} T_{l}\left(u_{1}\right)-D_{i} T_{l}\left(u_{2}\right)\right]\right\} d x+ \\
+\int_{E^{\prime \prime}}\left\{\sum_{i=1}^{n} a_{i}\left(x, \nabla T_{l}\left(u_{1}\right)\right)\left[D_{i} T_{l}\left(u_{1}\right)-D_{i} v\right]\right\} d x .
\end{gathered}
$$

From this fact, (21), and (4) it follows that

$$
\begin{gathered}
\int_{\Omega}\left\{\sum_{i=1}^{n} a_{i}\left(x, \nabla T_{l}\left(u_{1}\right)\right) D_{i} T_{k}\left(u_{1}-w\right)\right\} d x \geqslant \\
\geqslant \int_{G}\left\{\sum_{i=1}^{n} a_{i}\left(x, \nabla T_{l}\left(u_{1}\right)\right)\left[D_{i} T_{l}\left(u_{1}\right)-D_{i} T_{l}\left(u_{2}\right)\right]\right\} d x-\int_{\left(E^{\prime} \backslash G\right) \cup E^{\prime \prime}} g_{2} d x- \\
-\int_{E^{\prime} \backslash G}\left\{\sum_{i=1}^{n}\left|a_{i}\left(x, \nabla T_{l}\left(u_{1}\right)\right)\right|\left|D_{i} T_{l}\left(u_{2}\right)\right|\right\} d x-\int_{E^{\prime \prime}}\left\{\sum_{i=1}^{n}\left|a_{i}\left(x, \nabla T_{l}\left(u_{1}\right)\right)\right|\left|D_{i} v\right|\right\} d x .
\end{gathered}
$$


We denote by $I^{\prime}$ and $I^{\prime \prime}$ the third and fourth integral in the right-hand side of the latter estimate correspondingly. Using Young's inequality and (3), we get

$$
\begin{gathered}
I^{\prime} \leqslant c_{1} \int_{E^{\prime} \backslash G}\left\{\sum_{i=1}^{n} \nu_{i}\left|D_{i} T_{l}\left(u_{1}\right)\right|^{q_{i}}\right\} d x+\int_{E^{\prime} \backslash G}\left\{\sum_{i=1}^{n} \nu_{i}\left|D_{i} T_{l}\left(u_{2}\right)\right|^{q_{i}}\right\} d x+\int_{E^{\prime} \backslash G} g_{1} d x, \\
I^{\prime \prime} \leqslant c_{1} \int_{E^{\prime \prime}}\left\{\sum_{i=1}^{n} \nu_{i}\left|D_{i} T_{l}\left(u_{1}\right)\right|^{q_{i}}\right\} d x+\int_{E^{\prime \prime}}\left\{\sum_{i=1}^{n} \nu_{i}\left|D_{i} v\right|^{q_{i}}\right\} d x+\int_{E^{\prime \prime}} g_{1} d x .
\end{gathered}
$$

In view of Proposition 1, inclusions (22) and (23), and inequality (19) we have

$$
\begin{gathered}
\int_{\left(E^{\prime} \backslash G\right) \cup E^{\prime \prime}}\left\{\sum_{i=1}^{n} \nu_{i}\left|D_{i} T_{l}\left(u_{1}\right)\right|^{q_{i}}\right\} d x= \\
=\int_{\left(E^{\prime} \backslash G\right) \cup E^{\prime \prime}}\left\{\sum_{i=1}^{n} \nu_{i}\left|\delta_{i} u_{1}\right|^{q_{i}}\right\} d x \leqslant c_{3} k \int_{\left\{\left|u_{1}-v\right| \geqslant h-k\right\}} \Phi d x, \\
\int_{E^{\prime} \backslash G}\left\{\sum_{i=1}^{n} \nu_{i}\left|D_{i} T_{l}\left(u_{2}\right)\right|^{q_{i}}\right\} d x=\int_{E^{\prime} \backslash G}\left\{\sum_{i=1}^{n} \nu_{i}\left|\delta_{i} u_{2}\right|^{q_{i}}\right\} d x \leqslant c_{3} k \int_{\left\{\left|u_{2}-v\right| \geqslant h-k\right\}} \Phi d x .
\end{gathered}
$$

From (28)-(31) and (22), (23) we infer that

$$
\int_{\left(E^{\prime} \backslash G\right) \cup E^{\prime \prime}} g_{2} d x+I^{\prime}+I^{\prime \prime} \leqslant c_{4} k\left\{\int_{\left\{\left|u_{1}-v\right| \geqslant h-k\right\}} \Phi_{1} d x+\int_{\left\{\left|u_{2}-v\right| \geqslant h-k\right\}} \Phi_{2} d x\right\} .
$$

Using this inequality and (27), we deduce that

$$
\begin{gathered}
\int_{\Omega}\left\{\sum_{i=1}^{n} a_{i}\left(x, \nabla T_{l}\left(u_{1}\right)\right) D_{i} T_{k}\left(u_{1}-w\right)\right\} d x \geqslant \\
\geqslant \int_{G}\left\{\sum_{i=1}^{n} a_{i}\left(x, \nabla T_{l}\left(u_{1}\right)\right)\left[D_{i} T_{l}\left(u_{1}\right)-D_{i} T_{l}\left(u_{2}\right)\right]\right\} d x- \\
-c_{4} k\left\{\int_{\left\{\left|u_{1}-v\right| \geqslant h-k\right\}} \Phi_{1} d x+\int_{\left\{\left|u_{2}-v\right| \geqslant h-k\right\}} \Phi_{2} d x\right\} .
\end{gathered}
$$

In view of Proposition $1 \nabla T_{l}\left(u_{j}\right)=\delta u_{j}$ a.e. in $G, j=1,2$.

This fact, (32) and (20) imply

$$
\begin{gathered}
\int_{G}\left\{\sum_{i=1}^{n} a_{i}\left(x, \delta u_{1}\right)\left[\delta_{i} u_{1}-\delta_{i} u_{2}\right]\right\} d x \leqslant \\
\leqslant \int_{G_{1}} F\left(x, u_{1}\right) T_{k}\left(u_{1}-u_{2}\right) d x+c_{5} k\left\{\int_{\left\{\left|u_{1}-v\right| \geqslant h-k\right\}} \Phi_{1} d x+\int_{\left\{\left|u_{2}-v\right| \geqslant h-k\right\}} \Phi_{2} d x\right\} .
\end{gathered}
$$

By analogy we have

$$
\begin{gathered}
\int_{G}\left\{\sum_{i=1}^{n} a_{i}\left(x, \delta u_{2}\right)\left[\delta_{i} u_{2}-\delta_{i} u_{1}\right]\right\} d x \leqslant \\
\leqslant \int_{G_{1}} F\left(x, u_{2}\right) T_{k}\left(u_{2}-u_{1}\right) d x+c_{5} k\left\{\int_{\left\{\left|u_{1}-v\right| \geqslant h-k\right\}} \Phi_{1} d x+\int_{\left\{\left|u_{2}-v\right| \geqslant h-k\right\}} \Phi_{2} d x\right\} .
\end{gathered}
$$


Adding two latter inequalities, we establish that for every $k \geqslant 1, h \geqslant k+1$,

$$
\begin{gathered}
\int_{\left\{\left|u_{1}-u_{2}\right|<k,\left|u_{1}-v\right|<h,\left|u_{2}-v\right|<h\right\}}\left\{\sum_{i=1}^{n}\left[a_{i}\left(x, \delta u_{1}\right)-a_{i}\left(x, \delta u_{2}\right)\right]\left[\delta_{i} u_{1}-\delta_{i} u_{2}\right]\right\} d x \leqslant \\
\leqslant \int_{\left\{\left|u_{1}-v\right|<h,\left|u_{2}-v\right|<h\right\}}\left[F\left(x, u_{1}\right)-F\left(x, u_{2}\right)\right] T_{k}\left(u_{1}-u_{2}\right) d x+ \\
+2 c_{5} k\left\{\int_{\left\{\left|u_{1}-v\right| \geqslant h-k\right\}} \Phi_{1} d x+\int_{\left\{\left|u_{2}-v\right| \geqslant h-k\right\}} \Phi_{2} d x\right\} .
\end{gathered}
$$

As for a. e. $x \in \Omega$ the function $F(x, \cdot)$ is nonincreasing on $\mathbb{R}$, we have

$$
\left[F\left(x, u_{1}\right)-F\left(x, u_{2}\right)\right] T_{k}\left(u_{1}-u_{2}\right) \leqslant 0 \quad \text { a.e. in } \Omega .
$$

From Lemma 1 it follows that

$$
\text { meas }\left\{\left|u_{j}-v\right| \geqslant h-k\right\} \rightarrow 0, h \rightarrow+\infty, k \geqslant 1, j=1,2 .
$$

This fact imply

$$
\forall k \geqslant 1 \quad \int_{\left\{\left|u_{j}-v\right| \geqslant h-k\right\}} \Phi_{j} d x \rightarrow 0, \quad h \rightarrow+\infty, j=1,2 .
$$

Taking into account (34), (5), and using Fatou's lemma, we infer from (33), (35)

$$
\delta u_{1}=\delta u_{2} \quad \text { a. e. in } \Omega .
$$

Let again $k \geqslant 1, h \geqslant k+1$. Put

$$
z=T_{h}\left(u_{1}-v\right)-T_{h}\left(u_{2}-v\right) .
$$

Clearly, $z \in \stackrel{\circ}{W} \stackrel{1}{1, q}(\nu, \Omega)$. Hence, $T_{k}(z) \in \stackrel{\circ}{W^{1, q}}(\nu, \Omega)$. In view of Proposition 3 and Young's inequality we have

$$
\left(\int_{\Omega}\left|T_{k}(z)\right|^{n /(n-1)} d x\right)^{(n-1) / n} \leqslant c_{0} \sum_{i=1}^{n}\left(\int_{\Omega} \nu_{i}\left|D_{i} T_{k}(z)\right|^{q_{i}} d x\right)^{1 / q_{i}} .
$$

Let

$$
\begin{array}{ll}
H_{1}=\left\{|z|<k,\left|u_{1}-v\right|<h,\left|u_{2}-v\right|<h\right\}, & H_{2}=\left\{|z|<k,\left|u_{1}-v\right|<h,\left|u_{2}-v\right| \geqslant h\right\}, \\
H_{3}=\left\{|z|<k,\left|u_{1}-v\right| \geqslant h,\left|u_{2}-v\right|<h\right\}, & H_{4}=\left\{|z|<k,\left|u_{1}-v\right| \geqslant h,\left|u_{2}-v\right| \geqslant h\right\} .
\end{array}
$$

It is obvious that

$$
H_{m} \cap H_{r}=\varnothing, \quad m \neq r, \quad m, r=1, \ldots, 4, \quad\{|z|<k\}=\bigcup_{m=1}^{4} H_{m} .
$$

We fix an arbitrary $i \in\{1, \ldots, n\}$. Taking into account (2) and (38), we obtain

$$
\int_{\Omega} \nu_{i}\left|D_{i} T_{k}(z)\right|^{q_{i}} d x=\sum_{m=1}^{4} \int_{H_{m}} \nu_{i}\left|D_{i} z\right|^{q_{i}} d x .
$$


From Proposition 2 and (36) we get

$$
D_{i} z=0 \text { a.e. in } H_{1} \text {. }
$$

It is easy to show that

$$
H_{2} \subset\left\{h-k<\left|u_{1}-v\right|<h\right\}, \quad H_{3} \subset\left\{h-k<\left|u_{2}-v\right|<h\right\} .
$$

Besides, in view of Propositions 1 and 2,

$$
\begin{gathered}
D_{i} z=\delta_{i} u_{1}-D_{i} v \text { a.e. in } H_{2} \\
D_{i} z=D_{i} v-\delta_{i} u_{2} \quad \text { a.e. in } H_{3} .
\end{gathered}
$$

Using (41)-(43) and (19), we establish

$$
\begin{aligned}
& \int_{H_{2}} \nu_{i}\left|D_{i} z\right|^{q_{i}} d x \leqslant 2^{n}\left(c_{3}+1\right) k \int_{\left\{\left|u_{1}-v\right| \geqslant h-k\right\}} \Phi_{1} d x, \\
& \int_{H_{3}} \nu_{i}\left|D_{i} z\right|^{q_{i}} d x \leqslant 2^{n}\left(c_{3}+1\right) k \int_{\left\{\left|u_{2}-v\right| \geqslant h-k\right\}} \Phi_{2} d x .
\end{aligned}
$$

Finally, Propositions 2 and 1 imply that

$$
D_{i} z=0 \quad \text { a.e. in } H_{4} .
$$

From (39), (40), and (44)-(46) we deduce

$$
\int_{\Omega} \nu_{i}\left|D_{i} T_{k}(z)\right|^{q_{i}} d x \leqslant 2^{n}\left(c_{3}+1\right) k\left\{\int_{\left\{\left|u_{1}-v\right| \geqslant h-k\right\}} \Phi_{1} d x+\int_{\left\{\left|u_{2}-v\right| \geqslant h-k\right\}} \Phi_{2} d x\right\} .
$$

From this fact and (37) it follows that for every $k \geqslant 1$ and $h \geqslant k+1$,

$$
\begin{aligned}
& \left(\int_{\left\{\left|u_{1}-u_{2}\right|<k,\left|u_{1}-v\right|<h,\left|u_{2}-v\right|<h\right\}}\left|u_{1}-u_{2}\right|^{n /(n-1)} d x\right)^{(n-1) / n} \leqslant \\
\leqslant & c_{0} c_{6} k \sum_{i=1}^{n}\left\{\int_{\left\{\left|u_{1}-v\right| \geqslant h-k\right\}} \Phi_{1} d x+\int_{\left\{\left|u_{2}-v\right| \geqslant h-k\right\}} \Phi_{2} d x\right\}^{1 / q_{i}} .
\end{aligned}
$$

The latter result and assertion (35) allow to conclude that for every $k \geqslant 1$,

$$
\int_{\left\{\left|u_{1}-u_{2}\right|<k\right\}}\left|u_{1}-u_{2}\right|^{n /(n-1)} d x=0 .
$$

Hence, $u_{1}=u_{2}$ a.e. in $\Omega$.

\section{REFERENCES}

1. Aharouch L., Azroul E., Benkirane A. Quasilinear degenerated equations with $L^{1}$ datum and without coercivity in perturbation terms// Electron. J. Qual. Theory Differ. Equ. - 2006. - V.19. - 18 p. 
2. Atik Y., Rakotoson J.-M. Local T-sets and degenerate variational problems. I// Appl. Math. Lett. 1994. - V.7, №4. - P. 49-53.

3. Bendahmane M., Karlsen K.H. Nonlinear anisotropic elliptic and parabolic equations in $R^{N}$ with advection and lower order terms and locally integrable data// Potential Anal. - 2005. - V.22, №3. - P. $207-227$.

4. Bénilan Ph., Boccardo L., Gallouët T., Gariepy R., Pierre M., Vazquez J.L. An $L^{1}$-theory of existence and uniqueness of solutions of nonlinear elliptic equations// Ann. Scuola Norm. Sup. Pisa Cl. Sci. (4). - 1995. - V.22, №2. - P. 241-273.

5. Boccardo L., Gallouët T. Nonlinear elliptic and parabolic equations involving measure data// J. Funct. Anal. - 1989. - V.87, №1. - P. 149-169.

6. Boccardo L., Gallouët T. Nonlinear elliptic equations with right hand side measures// Comm. Partial Differential Equations. - 1992. - V.17, №3-4. - P. 641-655.

7. Boccardo L., Gallouët T., Marcellini P. Anisotropic equations in $L^{1} / /$ Differential Integral Equations. 1996. - V.9, №1. - P. 209-212.

8. Cavalheiro A.C. Existence of entropy solutions for degenerate quasilinear elliptic equations// Complex Var. Elliptic Equ. - 2008. - V.53, №10. - P. 945-956.

9. Cirmi G.R. On the existence of solutions to non-linear degenerate elliptic equations with measures data// Ricerche Mat. - 1993. - V.42, №2. - P. 315-329.

10. Kinderlehrer D., Stampacchia G. An introduction to variational inequalities and their applications. M.: Mir, 1983. - 256 p. (in Russian)

11. Kovalevsky A.A., Gorban Yu.S. Degenerate anisotropic variational inequalities with $L^{1}$-data - Institute of Applied Mathematics and Mechanics of NAS of Ukraine, Donetsk, 2007, preprint № 2007.01. - 92 p. (in Russian)

12. Kovalevsky A.A., Gorban Yu.S. Degenerate anisotropic variational inequalities with $L^{1}-$ data// C. R. Math. Acad. Sci. Paris. - 2007. - V.345, №8. - P. 441-444.

13. Kovalevsky A.A., Gorban Yu.S. On T-solutions of degenerate anisotropic elliptic variational inequalities with $L^{1}$-data// Izv. Math. - 2011. - V.75, №1. - P. 101-160. (in Russian)

14. Kovalevsky A.A., Gorban Yu.S. Solvability of degenerate anisotropic elliptic second-order equations with $L^{1}$-data// Electron. J. Differential Equations. - 2013. - №167. - P. 1-17.

15. Kovalevsky A.A. Entropy solutions of Dirichlet problem for a class of nonlinear elliptic fourth order equations with $L^{1}$-right-hand sides// Izv. Math. - 2001. - V.65, №2. - P. 27-80. (in Russian)

16. Li F.Q. Nonlinear degenerate elliptic equations with measure data// Comment. Math. Univ. Carolin. 2007. - V.48, №4. - P. 647-658.

Donetsk National University

yuliya_gorban@mail.ru

Received 2.02.2016 